\title{
Nova proposta de treinamento e avaliação do uso de auxílios ópticos em portadores de visão subnormal
}

\author{
New proposal for training and evaluation of the use of optical aids in low vision patients
}

\author{
Silvana Terezinha Figueiredo Moya ${ }^{1}$ \\ Lenira Maria Lima de Carvalho \\ Luciene Chaves Fernandes ${ }^{3}$ \\ André Aguiar Oliveira ${ }^{4}$
}

\section{RESUMO}

Objetivo: Elaboração de uma nova seqüência de treinamento e avaliação no uso do auxílio óptico para portadores de baixa visão. Métodos: Construção de tabelas obedecendo a uma seqüência de complexidade lingüística, iniciando com palavras, seguindo com expressões, períodos e culminando com textos. Utilização do novo material em 32 portadores de retinose pigmentária, com acuidade visual entre 0,5 e 1,3 LogMAR (20/63 e 20/400), registrando as velocidades de leitura com e sem auxílio óptico. Resultados: Tabelas de palavras, expressões, períodos e textos de tamanhos variados, com impressão de letras pretas em fundo branco e letras brancas em fundo preto. Apresentação dos dados referentes à idade, sexo, acuidade visual, auxílio óptico empregado, tamanho da impressão lida, velocidades de leitura sem e com auxílio e número de sessões de treinamento. Conclusões: A leitura de textos constitui o melhor instrumento de avaliação qualitativa e quantitativa no uso dos auxílios ópticos. As tabelas propostas baseiam-se em uma complexidade lingüística crescente, tentando minimizar ao máximo os fatores não relacionados à visão. Constitui valioso instrumento na prática da visão subnormal, entretanto, o desempenho de leitura depende de fatores relacionados a cada paciente.

Descritores: Baixa visão/reabilitação; Auxiliares sensoriais; Leitura; Testes visuais
Trabalho realizado no Serviço de Visão subnormal do Hospital São Geraldo - Hospital das Clínicas da U.F.M.G Doutoranda em Oftalmologia pela Universidade Federal de Minas Gerais.

${ }^{2}$ Pedagoga do serviço de Visão subnormal do Hospital São Geraldo - Hospital das Clínicas da Universidade Federal de Minas Gerais.

${ }^{3}$ Responsável pelo serviço de Visão subnormal do Hospital São Geraldo - Hospital das Clínicas da Universidade Federal de Minas Gerais.

${ }^{4}$ Professor Adjunto do Departamento de Oftalmologia da Universidade Federal de Minas Gerais.

Endereço para correspondência: Rua Piauí, 650 Apto 11 - São Paulo (SP) CEP 01214-000.

Recebido para publicação em 28/07/2000

Aceito para publicação em 27/07/2001

\section{INTRODUÇ̃̃̃O}

"Uma pessoa com baixa visão é aquela que possui um comprometimento do seu funcionamento visual, mesmo após tratamento e/ou correção de erros refracionais comuns, e tem, no melhor olho, uma acuidade visual inferior a 20/60 até percepção de luz, ou um campo visual inferior a 10 graus do seu ponto de fixação, mas que utiliza ou é potencialmente capaz de utilizar a visão para planejamento e execução de uma tarefa"(1). Para que este potencial visual seja desenvolvido, é necessário um treinamento para melhor utilização da visão residual, realizado através de sessões para o aprendizado do uso dos auxílios ópticos e não ópticos.

Considerando a leitura um importante fator de inclusão social, econômico e cultural, compreende-se a busca dos pacientes pela reabilitação no uso das habilidades relacionadas à leitura. Os profissionais da área de baixa visão deparam-se constantemente com a escassez de um material para leitura, que além de atender às funções visuais, se estruturasse também em Lingüística: complexidade fonética, morfológica e sintática ${ }^{(2)}$.

Diante das dificuldades apresentadas, surge a necessidade da criação e elaboração de um material que preencha esta lacuna. O objetivo deste estudo é a construção de um novo material que permita o treinamento e a avaliação quantitativa e qualitativa do uso efetivo dos auxílios ópticos. 


\section{MÉTODOS}

Dentro do contexto da nova proposta de treinamento e avaliação dos auxílios ópticos frente à leitura, é importante a elaboração de um processo gradativo de treinamento que minimize as dificuldades relacionadas à mesma. Foram então construídas cinco tabelas seguindo uma complexidade lingüística crescente: palavras, expressões e períodos, além da utilização de textos.

As palavras foram divididas em quatro grupos. Na língua portuguesa o tipo de sílaba mais comum é aquele formando por consoante-vogal, como as palavras do primeiro grupo: ma-to, se-re-ni-da-de. Quanto mais as sílabas das palavras se afastarem desse padrão maior o grau de dificuldade ${ }^{(2)}$, como se pode analisar na seqüência dos grupos:

- consoante-vogal-consoante: len-te, cor-po;

- consoante-consoante-vogal: Bra-sil, flo-ra

- e finalmente os dígrafos, que são grupos de duas letras representando um único som: so-nho, fi-lho.

Outros critérios levados em consideração na escolha das palavras foram o comprimento (dissílabas, trissílabas e polissílabas) e a familiaridade. Cada uma das tabelas apresenta uma sequiência de diferentes palavras.

No quinto e sexto grupos, utilizou-se expressões e períodos que fizessem parte do dia-a-dia do paciente. $\mathrm{O}$ treinamento foi concluído com textos, adaptados das fábulas de Ésopo $^{(3)}$, considerando o processamento lexical e sintático.

A impressão seguiu o sistema métrico, sendo cada tabela em um dos seguintes tamanhos 5M, 4M, 2,5M, 1,6M, e 1M, de acordo com a distância padrão de leitura de $40 \mathrm{~cm}$, com equivalência de Snellen de 20/250, 20/200, 20/125, 20/80 e 20/50. Esta variação pode ser ampliada usando distâncias maiores ou menores. Por definição da unidade métrica, $1 \mathrm{M}$ corresponde a letras com 5 segundos de arco a 1 metro, sendo o tamanho da letra $1 \mathrm{M}$ o de $1,45 \mathrm{~mm}$ em caixa baixa ${ }^{(4)}$. O espaço entre as linhas foi equivalente à altura das letras em caixa baixa ${ }^{(5)}$. O padrão de impressão utilizado foi o Times Roman, realizada em alto contraste $^{(4)}(100 \%)$, e em papel couchet fosco, 160 gramas. Na confecção do material, foi considerado o efeito da polaridade do contraste, ou seja, letras brancas impressas em fundo preto e letras pretas impressas em fundo branco, também com nível de contraste de $100 \%$.

Foram impressas dezenove fábulas de Ésopo sendo que cinco no tamanho 4M (20/200), seis em 2M (20/100) e oito em $1 \mathrm{M}(20 / 50)$, seguindo as características acima descritas, permitindo a determinação objetiva do desempenho da leitura através da medida do número de palavras corretamente lidas por minuto (velocidade de leitura), registradas em cronômetro.

A aplicabilidade do novo material, em projeto piloto, foi testada com trinta e dois pacientes portadores de retinose pigmentária, apresentado acuidade visual entre 0,5 (20/63) e 1,3 LogMAR (20/400).

Os pacientes participaram de sessões de treinamento com e sem auxílios ópticos, com a determinação das respectivas velocidades de leitura. Os auxílios ópticos empregados consistiram de óculos $(+3,0 ;+4,0 \mathrm{D} ;+5,0 \mathrm{D} ;+6,0 \mathrm{D} ;+8,0 \mathrm{D} ;+10,0 \mathrm{D}$; +12,0D; +16,0D; +20,0D), lupas de mão (2X; 3X; 4X; 5X), lupas de mesa $(2 \mathrm{X} ; 3 \mathrm{X} ; 4 \mathrm{X})$ e telessistema para perto binocular de $3 \mathrm{X}$.

\section{RESULTADOS}

\section{I - Material elaborado:}

Foram confeccionados duas apostilas para treinamento e avaliação do uso dos auxílios ópticos, constituídos por tabelas apresentando palavras, expressões, períodos e textos conforme as figuras 1, 2 e 3 . Alguns exemplos são mostrados abaixo:

\section{A) Palavras isoladas:}

\section{1 orupo}

mapa luxo figo vela foto

$2^{\circ}$ grupo

jantar rosto barco pacto leitor

$3^{\circ}$ grupo

prece tribo plantar graça prova

$4^{\circ}$ grupo

folha lenha ilha osso senha

B) Expressões:

5 grupo

escola de samba, futebol de areia, imposto de renda

C) Períodos:

6o grupo

Muitos animais como cão, gato e touro só enxergam bran co e preto, assim a capa vermelha do toureiro é uma farsa.

D) Textos:

"O leão e o mosquito"

Um leão ficou com raiva de um mosquito que não parava de zumbir ao redor de sua cabeça, mas o mosquito não deu a mínima.

- Você está achando que eu vou ficar com medo de você só porque você pensa que é rei? - disse ele altivo, e em seguida voou para o leão e deu uma picada ardida no seu focinho...

Todo o material acima descrito foi confeccionado em dupla

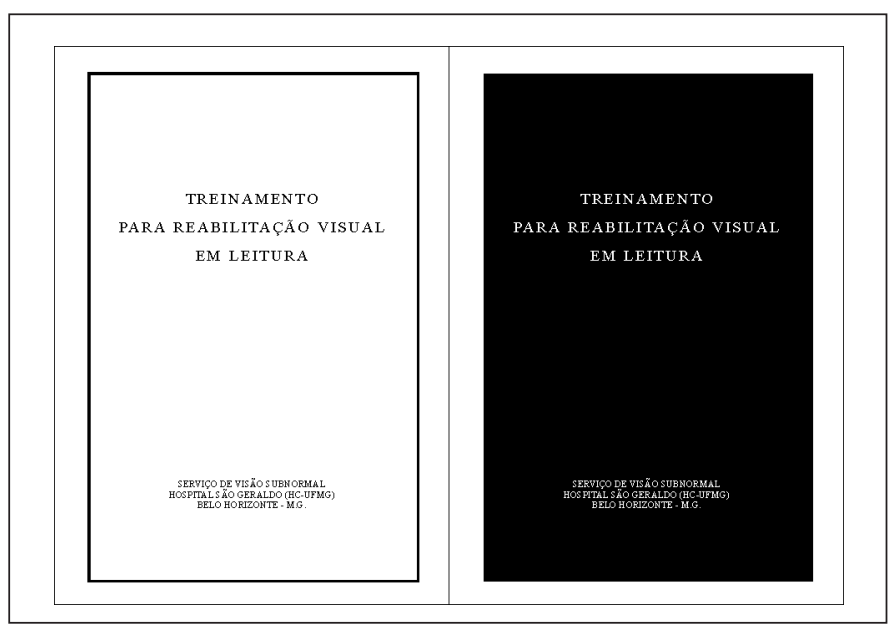

Figura 1 - Cadernos de treinamento contendo as diferentes tabelas 


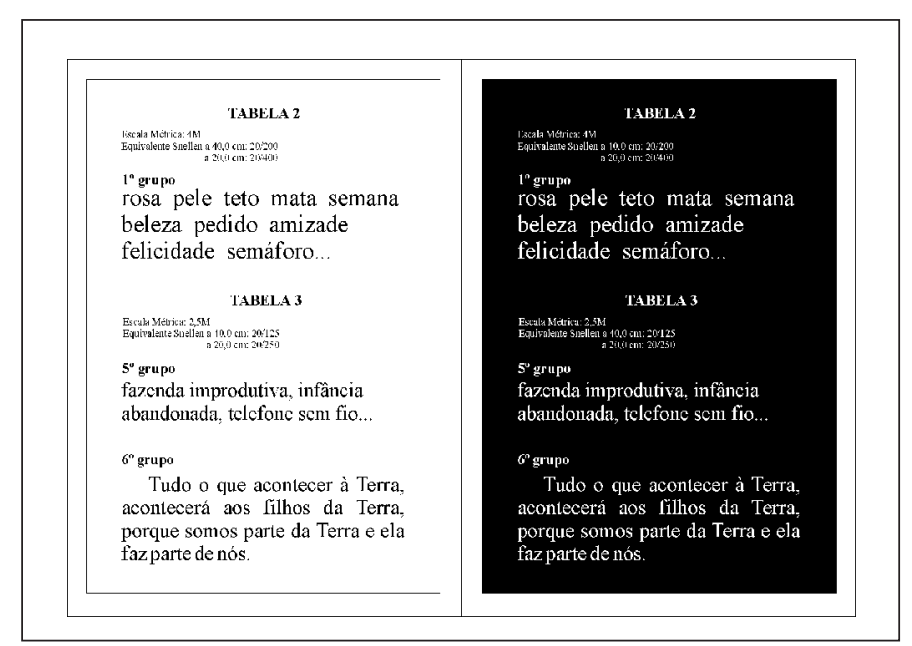

Figura 2 - Tabelas apresentando palavras, expressões e períodos de diferentes tamanhos e dupla polaridade de contraste

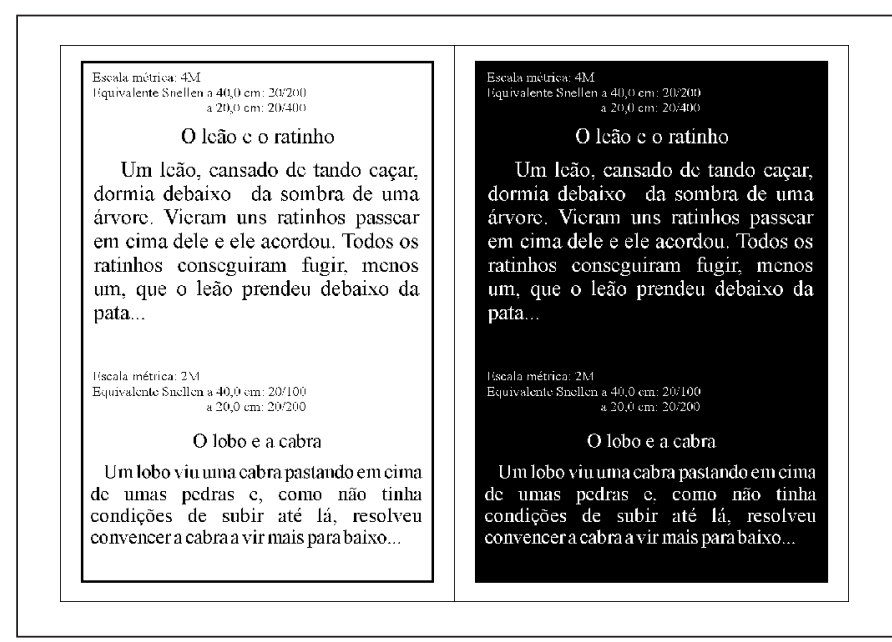

Figura 3 - Textos com variabilidade de tamanhos e impressão de letras pretas em fundo branco e letras brancas em fundo preto

polaridade (letras pretas em fundo branco e letras brancas em fundo preto).

\section{II - Aplicação das tabelas:}

Durante as sessões de treinamento, que variaram de 3 a 10 , os pacientes demonstraram facilidade no manuseio do material, familiaridade com as palavras e maior interesse pelo treinamento quando atingiam a leitura de textos.

Os resultados referentes ao novo material proposto são apresentados no Quadro 1.

\section{DISCUSSÃO}

O grande desafio desta discussão será responder às seguintes questões:

1 - Como medir objetivamente a adaptação ao auxílio óptico?

2 - Como elaborar um novo material para treinamento, já que as opções são poucas, principalmente em língua portuguesa?

A opção por leitura baseia-se em estudos psicolingüísticos que descrevem as habilidades oculares e perceptivas envolvidas neste processo( ${ }^{(6)}$. Para se chegar a esta opção é necessário que se entenda o que significa o ato de ler:

"Leitura é um processo complexo que envolve desde a percepção de sinais gráficos e sua tradução em som e imagem mental até a transformação dessa percepção em idéias provocando a geração de inferências, analogias, questionamentos e generalizações"(2). A percepção de sinais gráficos está ligada aos movimentos oculares: o olho não escorrega sobre o texto, mas salta rápida e irregularmente em todas as direções, com pausas para a fixação( ${ }^{(6)}$.

Analisando então o conceito de leitura e as complexidades inerentes ao processo, a leitura de texto para treinamento e avaliação da visão subnormal, surge como estratégia capaz de determinar quantitativa (velocidade de leitura) e qualitativamente a adaptação no uso dos auxílios ópticos ${ }^{(7)}$. O uso de tabelas com palavras isoladas pode fornecer a medida da acuidade de leitura, mas não a eficiência de leitura ${ }^{(4)}$.

As fábulas de Ésopo foram escolhidas para as tabelas de textos. São curtas, bem humoradas, populares e geralmente de fácil compreensão. Algumas, entretanto, foram adaptadas para que o vocabulário se tornasse acessível à maioria dos pacientes, tentando minimizar ao máximo as dificuldades cognitivas e lingüísticas, não relacionadas à visão.

Na prática clínica dados como medida da acuidade visual, biomicroscopia (transparência ou opacidades dos meios) e fundoscopia (localização e extensão de lesões), não são suficientes para predizer o desempenho em leitura ${ }^{(8-10)}$. Portanto, o uso de tabelas de texto poderá fornecer dados complementares sobre movimentos oculares, existência de escotomas e diminuição na sensibilidade ao contraste ${ }^{(9-12)}$. Essas tabelas possuem ainda ampla aplicabilidade clínica permitindo avaliar as mudanças no desempenho da leitura após treinamento, estimar o potencial de leitura com o auxílio óptico e orientar na prescrição desses auxílios ${ }^{(10)}$.

Uma das principais preocupações na elaboração deste novo material foi embasar-se nos princípios lingüísticos da língua portuguesa, o que norteou a sequiência das tabelas impedindo, portanto, a tradução de materiais similares existentes em outras línguas ${ }^{(11)}$. Considerou-se também a magnificação linear, padrão de impressão e a polaridade do contraste. O padrão escolhido foi o Times Roman, por aproximar-se do estilo e da legibilidade dos tipos de impressão mais comumente utilizados em jornais e livros ${ }^{(4)}$.

Legge et al. ${ }^{(12)}$, em estudo a respeito dos fatores visuais que interferem na leitura de pacientes com visão normal concluíram que, para estes pacientes, o desemprenho da leitura não é influenciado pela mudança da polaridade do contraste, o mesmo não podendo ser afirmado a respeito dos portadores de visão subnormal. Alguns pacientes com baixa visão apresentam melhora na eficiência da leitura diante da impressão de letras brancas em fundo preto, preferência atribuída à disper- 


\begin{tabular}{|c|c|c|c|c|c|c|c|}
\hline \multicolumn{8}{|c|}{ Quadro 1. Dados relacionados à aplicabilidade das tabelas de treinamento } \\
\hline Paciente & $\begin{array}{l}\text { Idade } \\
\text { (anos) }\end{array}$ & Sexo & $\begin{array}{l}\text { AV } \\
\text { (M) }\end{array}$ & Auxílio óptico & Impressão & $\begin{array}{c}\text { VL } \\
\text { (palavras/min) }\end{array}$ & $\begin{array}{l}\text { Sessões } \\
\text { (número) }\end{array}$ \\
\hline 1 & 27 & $\mathrm{~F}$ & 1,25 & óculos +3,0D & $1,0 \mathrm{M}$ & $160 / 120$ & 3 \\
\hline 2 & 38 & M & 1,6 & óculos $+4,0 \mathrm{D}$ & $1,0 \mathrm{M}$ & $0 / 37$ & 6 \\
\hline 3 & 34 & M & 2,0 & óculos +5,0D & $1,0 \mathrm{M}$ & $0 / 115$ & 3 \\
\hline 4 & 43 & $F$ & 2,5 & óculos +6,0D & $1,0 \mathrm{M}$ & $0 / 101$ & 4 \\
\hline 5 & 55 & $\mathrm{~F}$ & 1,6 & óculos +4,0D & $1,0 \mathrm{M}$ & $0 / 50$ & 6 \\
\hline 6 & 23 & $M$ & 2,5 & lupa mesa $2 X$ & $1,0 \mathrm{M}$ & $86 / 65$ & 7 \\
\hline 7 & 32 & $M$ & 4,0 & lupa mesa $3 X$ & $1,0 \mathrm{M}$ & $0 / 58$ & 3 \\
\hline 8 & 62 & $F$ & 2,0 & óculos +5,0D & $1,0 \mathrm{M}$ & $41 / 35$ & 8 \\
\hline 9 & 36 & $M$ & 2,5 & lupa mesa 2X & $1,0 \mathrm{M}$ & $0 / 62$ & 7 \\
\hline 10 & 17 & $\mathrm{~F}$ & 1,25 & óculos +3,0D & $1,0 \mathrm{M}$ & $125 / 128$ & 3 \\
\hline 11 & 34 & $\mathrm{~F}$ & 1,6 & óculos +4,0D & $1,0 \mathrm{M}$ & $135 / 133$ & 3 \\
\hline 12 & 14 & $\mathrm{~F}$ & 6,4 & óculos $+16,0 \mathrm{D}$ & $1,0 \mathrm{M}$ & $0 / 56$ & 4 \\
\hline 13 & 40 & M & 1,6 & óculos +4,0D & $1,0 \mathrm{M}$ & $0 / 67$ & 5 \\
\hline 14 & 44 & $M$ & 2,5 & lupa mão 2X & $1,0 \mathrm{M}$ & $0 / 58$ & 6 \\
\hline 15 & 68 & $M$ & 1,25 & óculos +3,0D & $1,0 \mathrm{M}$ & $0 / 50$ & 6 \\
\hline 16 & 49 & $\mathrm{~F}$ & 3,2 & óculos +8,0D & $1,0 \mathrm{M}$ & $0 / 48$ & 6 \\
\hline 17 & 26 & $\mathrm{~F}$ & 6,4 & lupa mesa $4 X$ & $1,0 \mathrm{M}$ & $0 / 68$ & 9 \\
\hline 18 & 18 & $\mathrm{~F}$ & 5,0 & TS $3 X$ & $1,0 \mathrm{M}$ & $0 / 82$ & 8 \\
\hline 19 & 32 & $\mathrm{~F}$ & 1,6 & óculos +4,0D & $1,0 \mathrm{M}$ & 0/99 & 4 \\
\hline 20 & 28 & $M$ & 6,4 & óculos $+16,0 \mathrm{D}$ & $2,0 \mathrm{M}$ & $0 / 29$ & 7 \\
\hline 21 & 20 & $\mathrm{~F}$ & 4,0 & lupa mão 2X & $1,0 \mathrm{M}$ & $0 / 25$ & 6 \\
\hline 22 & 42 & $M$ & 8,0 & óculos $+20,0 \mathrm{D}$ & $2,0 \mathrm{M}$ & $0 / 30$ & 7 \\
\hline 23 & 12 & $M$ & 6,4 & lupa mesa 4X & $4,0 \mathrm{M}$ & $0 / 7$ & 6 \\
\hline 24 & 19 & $\mathrm{~F}$ & 8,0 & óculos $+20,0 \mathrm{D}$ & $4,0 \mathrm{M}$ & $0 / 16$ & 10 \\
\hline 25 & 32 & $\mathrm{~F}$ & 2,5 & óculos +6,0D & $1,0 \mathrm{M}$ & $0 / 28$ & 8 \\
\hline 26 & 08 & $\mathrm{~F}$ & 1,6 & óculos +4,0D & $1,0 \mathrm{M}$ & $33 / 24$ & 6 \\
\hline 27 & 30 & $M$ & 6,4 & óculos $+16,0 \mathrm{D}$ & $2,0 \mathrm{M}$ & $0 / 64$ & 7 \\
\hline 28 & 23 & $M$ & 6,4 & óculos $+16,0 \mathrm{D}$ & $2,0 \mathrm{M}$ & $0 / 46$ & 8 \\
\hline 29 & 28 & $\mathrm{~F}$ & 8,0 & óculos $+20,0 \mathrm{D}$ & $2,0 \mathrm{M}$ & $0 / 46$ & 8 \\
\hline 30 & 46 & $M$ & 2,5 & óculos +6,0D & $2,0 \mathrm{M}$ & $0 / 26$ & 7 \\
\hline 31 & 26 & $M$ & 8,0 & óculos $+20,0 \mathrm{D}$ & $2,0 \mathrm{M}$ & $0 / 35$ & 9 \\
\hline 32 & 21 & $\mathrm{~F}$ & 8,0 & óculos $+20,0 \mathrm{D}$ & $1,0 \mathrm{M}$ & $0 / 21$ & 7 \\
\hline
\end{tabular}

são anormal da luz em olhos com opacidades dos meios. Medidas fotométricas indicam que na impressão de letras pretas em fundo branco mais de $80 \%$ da luz é refletida ${ }^{(9,13)}$.

Com aplicação do material, contatou-se o que, até o momento, foi enfatizado a respeito do valor de um material baseado na lingüística do país em que é empregado. Neste estudo, o novo material proporcionou:

- padronização do treinamento;

- critérios para avaliação quantitativa e qualitativa do desempenho de leitura;

- boa aceitabilidade pelo paciente.

Dados que comprovam, portanto, a sua utilidade.

Materiais destinados à avaliação e treinamento de pacientes com baixa visão, impressos em inglês, como o MNREAD ${ }^{(11)}$ e o Pepper test ${ }^{(14)}$, encontram-se disponíveis para utilização clínica. No entanto, segundo a literatura pesquisada e disponível, não foi encontrado material similar, desenvolvido no Brasil, o que justifica a realização deste estudo.
O novo material constitui instrumento para o aperfeiçoamento da prática da visão subnormal, entretanto, os resultados também dependem de fatores relacionados a cada paciente.

\section{CONCLUSÕES}

1) A leitura mostrou-se um instrumento adequado para o treinamento e avaliação da adaptação aos auxílios ópticos para pacientes com visão subnormal, sendo a leitura de textos uma maneira que comporta, além do treinamento, avaliação quantitativa, através da medida de palavras lidas corretamente;

2) O material aqui desenvolvido é baseado na complexidade lingüística e nas dificuldades visuais, podendo orientar de maneira objetiva os especialistas da área;

3) A validação das tabelas poderá ser concluída com sua aplicação em um número significativo de pacientes, programação para um novo estudo. 


\section{ABSTRACT}

Purpose: The design and use of new near-vision charts for training and evaluation of the use of optical aids in low vision patients. Methods: Construction of new charts obeying a linguistic complexity, starting with words, followed by expressions and sentences and ending with texts. The new material was used in thirty-two patients with retinitis pigmentosa, with a corrected visual acuity from 0.5 to 1.3 LogMAR. Reading speeds were recorded with and without optical aids. Results: Word-reading, expression-reading, sentence-reading and textreading charts in different sizes, with black print on a white background and white print on a black background. The data regarding age, sex, visual acuity, optical aids, print size, reading speeds with and without aids and educational training are shown. Conclusion: Text reading is the best instrument in qualitative and quantitative evaluation of the use of optical aids. The proposed charts have an increasing linguistic complexity trying to minimize the nonvisual factors. This is an important material for low vision, but reading performance depends on features of each patient.

Keywords: Low vision/rehabilitation; Reading; Sensory aids; Vision tests

\section{REFERÊNCIAS}

1. World Health Organization. Management of low vision in children: report of a WHO consultation. Bangkok; julho, 1992. Geneva; 1993. 46 p.

2. Coscarelli CV. Leitura em ambiente multimídia e a produção de inferências [tese]. Belo Horizonte: Faculdade de Letras da Universidade Federal de Minas Gerais; 1999.

3. Ash R, Higton B. comp. Fábulas de Ésopo, 7ạa. São Paulo: Companhia das Letrinhas; 1997. 95 p.

4. Bailey IL, Lovie JE. The design and use of a new near-vision chart. Am J Optom Physiol Opt 1980;57:378-87.

5. Odutan AO, al-Abdulmunem MA. Design of an Arabic near visual acuity chart. Ophthalmic Physiol Opt 1997;17:58-60.

6. Fulgêncio L, Liberato YG. Como facilitar a leitura: repensando a Língua Portuguesa. 3a . São Paulo: Contexto;1998. cap. 1.

7. Nilsson UL. Visual rehabilitation with and without educational training in the use of optical aids and residual vision. A prospective sutdy of patients with advanced age-related macular degeneration. Clin Vis Sci 1990;6:3-10.

8. Legge GE, Ross JA, Isemberg LM, LaMay JM. Psychophysics of reading, XII. Clinical predictors of low-vision reading speed. Invest Ophthamol Vis Sci 1992;33:677-87.

9. Legge GE, Rubin GS, Pelli DG, Schleske MM. Psychophysics of reading II. Low vision. Vision Res 1985a;25:253-65.

10. Ahn SJ, Legge GE, Luebker A. Printed cards for measuring low-vision reading speed. Vision Res 1995;35:1939-44.

11. Legge GE, Ross JA, Luebker A, LaMay JM. Psychophysics of reading VIII. The Minnesota Low-Vision Reading Test. Optom Vis Sci 1989;66:843-53.

12. Legge GE, Pelli DG, Rubin GS, Schleske MM. Psychophysics of reading 1. Normal Vision. Vision Res 1985b;25:239-52.

13. Rubin GS, Legge GE. Psychophysics of reading VI - The role of contrast in low vision. Vision Res 1989;29:79-91.

14. Watson G, Baldasare J, Whittaker S. The validity and clinical use of the Pepper Visual Skills for reading test. J Impairment Blind 1990;84:119-23.

\title{
XIII Congresso da Sociedade Norte-Nordeste de Oftalmologia 15 a 18 de Maio de 2002 Maceió - AL
}

\author{
Informações: tel.: (82) 327-6859 \\ fax: (82) 231-1619 \\ e-mail: nnoftalmo.maceio@globo.com
}

